\title{
Contenido de sodio en productos alimenticios preenvasados $y$ comercializados en El Salvador
}

\author{
DOI 10.5377/alerta.v4i3.10954 \\ Yris Eugenia Ramos Flores \\ Región de Salud Metropolitana, Ministerio de Salud, San Salvador, El Salvador. \\ Correspondencia \\ $\checkmark$ ydea_2205@yahoo.com \\ (D) $0000-0003-2548-1560$
}

\begin{abstract}
Resumen
Introducción. La dieta actual de la población se caracteriza por la sustitución de alimentos naturales por productos alimenticios procesados, formulados industrialmente con ingredientes que contienen sodio. Objetivo. Clasificar el contenido de sodio en productos alimenticios preenvasados comercializados en El Salvador, según el perfil de nutrientes de la Organización Panamericana de la Salud y las metas regionales para formulación industrial. Metodología. Diseño transversal descriptivo, se analizaron las etiquetas nutricionales de 1016 productos de siete categorías, registrados en el Sistema de Información en Salud Ambiental. Para la clasificación del contenido de sodio se aplicaron fórmulas según el perfil de nutrientes: alto en sodio $\geq 1$ miligramo de sodio por caloría. Para la clasificación según metas regionales: cuando el contenido de sodio en 100 gramos de producto es > a la meta establecida. Resultados. Según el perfil de nutrientes, el $52 \%$ de los productos tiene alto contenido de sodio. Según metas regionales, el $24 \%$ está formulado por arriba de las metas, esto refleja diferencias entre las valoraciones de contenido de sodio por categoría. Conclusión. Más de la mitad los productos alimenticios preenvasados comercializados en El Salvador son clasificados como altos en sodio, según el perfil de nutrientes. Una cuarta parte de estos están formulados con sodio por encima de las metas regionales, mostrando que las metas son más flexibles en la valoración de contenido máximo de sodio para la formulación industrial en relación con la valoración de contenido alto en sodio para consumo humano.

Palabras clave

Sodio en la dieta, etiquetado nutricional, etiquetado de alimentos, ingredientes alimentarios.
\end{abstract}

\section{Abstract}

Introduction. The main characteristic of the population current diet is the consumption of industrial manufactured foods, substituting natural ingredients by processed ones, containing ingredients high in sodium. Objective. To classify prepackaged food products marketed in El Salvador, according to their sodium content, following the nutrients profile of Pan American Health Organization, and the regional goals for industrial manufacturing. Methodology. Descriptive crosssectional study. Nutritional labels were analyzed from 1016 products from seven categories registered in the Environmental Health Information System. For the classification of sodium content, formulas were applied according to the nutrients profiles: high in sodium >1 miligrams per calory; for the classification according to the regional goals: sodium content in 100 grams of the product is $>$ than the stablished goal. Results. According to nutrients profiles, $52 \%$ of all products presented a high sodium content. Whether, following the regional goals, $24 \%$ is manufactured surpassing the goals, reflecting sodium content differences by each category. Conclusion. Half the pre-packaged food marketed in El Salvador, are classified as high in sodium according to the nutrients profile, whereas a fourth of them are classified as containing sodium above the regional goals, showing a more flexible assessment of sodium contain for industrial manufacturing formulation than the assessment of high sodium content for human consumption.

Keywords

Sodium in diet, nutritional labels, food labels, food ingredients.

\section{G}

ACCESO ABIERTO

Sodium content on prepackaged food products marketed in El Salvador

\section{Citación recomendada:}

Ramos Flores YE. Contenido de sodio en productos alimenticios preenvasados comercializados en El Salvador. Alerta. 2021; 4(3):135-142.

DOI: $10.5377 /$ alerta.v4i3.10954

Recibido:

28 de febrero de 2021

\section{Aceptado:}

11 de julio de 2021

\section{Publicado:}

26 de julio de 202

Contribución de autoría: YERF': Diseño del protocolo, recolección y análisis de datos, elaboración del manuscrito.

\section{Conflicto de intereses:} No existe ningún tipo de conflicto de interés con el estudio por parte de la investigadora.

\section{Introducción}

Las enfermedades no transmisibles (ENT) son la principal causa de muerte a nivel mundial (71 \% de las muertes). Cada año producen la muerte de aproximadamen- te 41 millones de personas. Las ENT son el resultado de de la combinación de factores genéticos, fisiológicos, ambientales y conductuales. El $10 \%$ de estas muertes se relacionan con factores modificables como la ingesta excesiva de sodio!' 
El patrón de consumo alimentario en el mundo presenta dietas altas en alimentos procesados con ingredientes que adicionan a la dieta cantidades excesivas de sodio entre otros nutrientes críticos. El alto consumo de sodio en la población es uno de los tres principales factores de riesgo nutricionales en todo el mundo. A menor ingesta de sodio menor riesgo de enfermedad cardiovascular y mortalidad ${ }^{2}$. Estudios de excreción de sodio en orina han revelado que el consumo de sal de la población centroamericana está por encima de $5 \mathrm{~g}$ al día, mostrando que el consumo de sodio varía entre $3,5 \mathrm{~g}$ y 3,8 g, equivalente a $8,9 \mathrm{~g}$ a $9,8 \mathrm{~g}$ de sal por $\mathrm{dia}^{3}$.

La Organización Panamericana de la Salud (OPS/OMS) ha liderado esfuerzos regionales para reducir el consumo de sodio de la dieta en las Américas, impulsando la armonización de metas regionales de contenido de sodio en categorías de alimentos procesados, las cuales fueron acogidas en la estrategia regional para la reducción del consumo de sal y sodio en Centroamérica y República Dominicana 2019-2025, por el Consejo de Ministros de Salud de Centroamérica (COMISCA) 3,4. Además en el 2016, la OPS/OMS presentó el modelo de perfil de nutrientes, que tiene como finalidad proporcionar una herramienta para clasificar los alimentos y bebidas procesadas y ultraprocesados que contienen una cantidad excesiva de nutrientes críticos como el sodio ${ }^{5}$.

En 2017, las muertes por ENT en El Salvador representaron el $48 \%$ del total de muertes por todas las causa. Para el 2021 se considera que las ENT es uno de los mayores desafíos del Sistema Nacional Integrado de Salud, ya que de forma creciente contribuye a la mortalidad general y es la causa más frecuente de mortalidad prematura y discapacidad representando una gran carga económica sanitaria ${ }^{6,7}$.

La prevalencia de hipertensión arterial en la población salvadoreña es del $37 \%$ y se considera que el consumo de sal es elevado en el $12,8 \%$ de la población adulta, calculada únicamente por la frecuencia con la que la población agrega sal a la comida al momento de comer ${ }^{8}$.

El Salvador cuenta con un Reglamento Técnico Centroamericano de Etiquetado Nutricional de carácter voluntario. Al presentar la etiqueta nutricional se declara el contenido de sodio que tienen los productos alimenticios y no existe una clasificación que interprete el nivel de sodio o clasifique el contenido alto en sodio de los productos alimenticios preenvasados que se consumen en el país. Se cuenta con un registro sanitario obligatorio que se consigna en el Sistema de Información de Salud Ambiental
(SISAM) del Ministerio de Salud (MINSAL), a través del módulo de gestión de alimentos y bebidas, encontrándose la información de la etiqueta nutricional de los productos que la declaran. Asimismo, cada día el MINSAL autoriza el registro sanitario de nuevos productos para ser comercializados en todo el país. El presente estudio tiene como objetivo clasificar el contenido de sodio en productos alimenticios preenvasados comercializados en El Salvador, según el perfil de nutrientes OPS/OMS y las metas regionales para formulación industrial

\section{Metodología}

Estudio con un diseño transversal descriptivo. La unidad de análisis fueron las etiquetas nutricionales de productos alimenticios registrados en el SISAM, en el periodo del 2 de mayo al 30 de noviembre del 2019.

Se incluyeron 1016 productos alimenticios preenvasados de siete categorias categorías: pan, galletas, carnes, cereales de desayuno, snack, pastas y sopas correspondientes a todos los productos que cumplían con los criterios de inclusión (registro sanitario aprobado, procesados y ultraprocesados, con etiqueta nutricional legible y conforme a sus ingredientes). Se excluyeron el pescado y mariscos por no ser ultaprocesados, además el snack dulce, pasteles, tortas, bizcochos, carnes secas curadas y carnes conservadas a temperatura ambiente, pastas y fideos de larga duración secos y crudos y fideos en caldo. Para el estudio se excluyeron las categorías: mayonesa, condimentos, mantequillas y lácteos de untar por ser utilizados como ingredientes y no ser productos listos para consumirlos (Tabla 1).

Para el análisis se tomaron en cuenta las siguientes variables: productos alimenticios, categorías de alimentos, país de procedencia, peso en gramo (g), calorías y sodio en miligramo (mg). Es importante hacer notar que cada categoría presenta grandes diferencias en pesos en sus multiples presentaciones comerciales, además que por su condición de procesados o ultra procesados, contienen distintos ingredientes, lo que imposobilita su comparación.

Los datos fueron obtenidos del SISAM en el módulo de gestión y control de alimentos y bebidas en la sección: «Solicitudes», subsección «Registro Sanitario», subsección «Bandeja de solicitudes», subsección «Aprobadas» en la cual se encuentran todos los productos registrados. Para esto se empleó una hoja electrónica en Excel, elaborada para fines del estudio y denominada «Instrumento de recolección de datos para clasificación del contenido de sodio en productos 
alimenticios preenvasados comercializados en El Salvador». Para la triangulación de los datos se contrastaron las etiquetas nutricionales con los ingredientes registrados en la etiqueta general de los productos alimenticios para revisar la congruencia o detección de declaraciones imprecisas.

Para la clasificación del contenido de sodio en los alimentos y fines del estudio se construyeron unas fórmulas en Excel con puntos de corte, según el perfil de nutrientes «alto en sodio: > 1 mg de sodio por caloría y contenido de sodio dentro de lo establecido $<1 \mathrm{mg}$ de sodio por caloría». Para la clasificación, según metas regionales «cuando el contenido de sodio en $100 \mathrm{~g}$ de producto es menor a la meta establecida por categoría de alimento y cumple la meta cuando el contenido de sodio en $100 \mathrm{~g}$ de producto es < a la meta establecida por categoría de alimento», según la estrategia regional para la reducción del consumo de sal y sodio en Centroamérica y República Dominicana. La meta regional de sodio para la formulación industrial en $100 \mathrm{~g}$ es, para el pan «600 mg», galletas «485 mg dulces y saladas 1340 mg», carnes «todo tipo cocinadas procesadas y salchichas 1210 mg y aves empanizada 735 mg», cereales de desayuno «630 mg», snack «900 mg», pastas y fideos de larga duración tal como se consumen «640 mg» y sopas como se consumen «360 mg».

En el análisis de los datos se obtuvieron porcentajes de los productos por país de procedencia (nacionales, Centroamérica y otros países), contenido promedio de sodio en mg, promedio de calorías en $100 \mathrm{~g}$ por cada categoría de producto y razón sodio caloría. Se aplicaron las fórmulas construidas para calcular si el contenido de sodio era alto, según perfil de nutrientes por producto y se obtuvieron los porcentajes de productos altos en sodio por categoría. Se calcularon los porcentajes de productos con contenido de sodio mayor a las metas regionales por categoría.

\section{Resultados}

El $73 \%$ de los productos alimenticios preenvasados que se comercializan en El Salvador se importan de 31 países, 13 países de la región de las Américas y 18 de Europa y Asia.

Respecto al pan, este procede de 8 países, las galletas de 25 , las carnes de 7, los cereales de desayuno de 6 , los snacks de 4, las sopas de 8 y las pastas de 7 países. En todas las categorías se encontraron productos de origen salvadoreño (269) y de otros países, principalmente de los Estados Unidos, (305) y México (113), que se presenta en la Tabla 1.
Tabla 1. Productos alimenticios preenvasados según procedencia.

\begin{tabular}{lrrrr}
\hline Categorías & Nacional & $\begin{array}{l}\text { Centro- } \\
\text { américa }\end{array}$ & \multicolumn{1}{c}{$\begin{array}{l}\text { Otros } \\
\text { países }\end{array}$} & Total \\
\hline Pan & 119 & 0 & 81 & 200 \\
Galletas & 56 & 0 & 344 & 400 \\
Carnes & 45 & 3 & 105 & 153 \\
Cereal & 8 & 1 & 116 & 125 \\
para el & & & & \\
desayuno & & & & \\
Snacks & 35 & 0 & 40 & 75 \\
Sopas & 1 & 0 & 34 & 35 \\
Pastas & 5 & 0 & 23 & 28 \\
Total & 269 & 4 & 743 & 1016 \\
\hline
\end{tabular}

Sobre la clasificación de los productos alimenticios preenvasados por su contenido de sodio, según perfil de nutrientes, se encontró que más de la mitad (52\%) se clasifican como «altos en sodio». Los porcentajes varían por categorías. El $100 \%$ de las sopas son altas en sodio más del $95 \%$ de las carnes y pastas son altos en sodio, seguidas de los snacks, pan, cereales de desayuno y galletas. Respecto a la meta regional de contenido de sodio en productos alimenticios, el $24 \%$ están formulados por arriba de las metas; así el $91 \%$ de las sopas y el $64 \%$ de las pastas contienen sodio por arriba de las metas regionales.

Estos resultados reflejan diferencias entrer la valoración de contenido de siodio según el perfil de nutrientes como referencia de consumo humano y la valoración según las metas regionales para la formulación industrial (Tabla 2).

Tabla 2. Clasificación de productos alimenticios preenvasados por su contenido de sodio ${ }^{4}$

\begin{tabular}{llll}
\hline Categoría & $\begin{array}{l}\geq \text { a } 1 \mathrm{mg} \\
\text { de sodio/ } \\
\text { caloría, \% }\end{array}$ & $\begin{array}{l}\text { Sodio > a la } \\
{ }^{*} \text { meta regional } \\
\text { en } 100 \mathrm{~g}, \%\end{array}$ & Total \\
\hline Pan & $54(108)$ & $26(52)$ & 200 \\
Galletas & $26(104)$ & $14(56)$ & 400 \\
Carnes & $97 /(148)$ & $36(55)$ & 153 \\
Cereal & $53(66)$ & $13(16)$ & 125 \\
para el & & & \\
desayuno & & & 75 \\
Snacks & $55(41)$ & $23(17)$ & 35 \\
Sopas & $100(35)$ & $91(32)$ & 28 \\
Pastas & $96(27)$ & $64(18)$ & 1016 \\
Total & $52(529)$ & $24(246)$ & \\
\hline
\end{tabular}

El número de productos $\geq$ a $1 \mathrm{mg}$ de sodio/ caloría y con sodio mayor a la meta regional está en paréntesis.

* La meta regional: pan (600 mg), galletas (485 mg) dulces y saladas (1340 mg), carnes procesadas (1210 mg) y empanizada (735 mg), cereales de desayuno (630 mg), snacks (900 mg), pastas (640 mg) y sopas (360 mg). 
Al evaluar el promedio de sodio por calorías en 100 gramos de cada producto alimenticio se encontró que las pastas, sopas y carnes son las que más contenido de sodio registran por calorías y solo la categoría galletas resulto con un promedio de sodio menor al promedio de calorías (Figura 1).

Para enfatizar el contenido de sodio por calorías que proveen cada una de las categorías de productos alimenticios en estudio, se expone la Figura 2.

Las categorías de productos alimenticios procesados que más se desvían de la relación sodio/calorías són: las pastas (5,6 más sodio por caloría), las sopas (5 veces mas sodio), las carnes (4,4 veces mas sodio), los snacks (1,4 veces mas sodio), el pan (1,2 veces mas sodio) y los cereales de desayuno 1,01 veces más sodio.

Estos resultados reflejan diferencias entre la valoración de contenido de sodio, según el perfil de nutrientes para el consumo humano y la valoración según las metas regionales para la formulación industrial.

Al evaluar el contenido de sodio por categoría específica se encontró que el $54 \%$ del pan resultó alto en sodio y el $26 \%$ está por arriba de la meta regional. El contenido promedio de sodio en el pan fue de 542 mg, y calorías 436 en 100 gramos.

El $26 \%$ de las galletas resultó alto en sodio, de éste 29 de 30 galletas saladas alto en sodio y 75 de 370 galletas dulces. El $14 \%$ del total de galletas están formuladas por arriba de las metas regionales, las cuales fueron evaluadas con dos tipos de metas: galletas dulces, $485 \mathrm{mg}$ de sodio, resultando 56 de 370 galletas dulces por arriba de la meta regional y las saladas ninguna de 30 por arriba de la meta regional de $1340 \mathrm{mg}$ de sodio en $100 \mathrm{~g}$ de producto. El contenido promedio de sodio en galletas fue de 370 mg, para dulces de $334 \mathrm{mg}$ y saladas $807 \mathrm{mg}$.

El $97 \%$ de las carnes resultó alto en sodio y el $36 \%$ está formulado por arriba de las metas regionales, siendo la meta de 1210 mg de sodio en $100 \mathrm{~g}$ en carnes cocinadas y carnes procesadas y salchichas, y en carnes y aves empanizadas de $735 \mathrm{mg}$ de sodio en 100 g. Se determinó el contenido de sodio promedio en carnes de 1104 mg para carnes cocinadas; para carnes crudas y procesadas y salchichas de $1143 \mathrm{mg}$ y de carnes y aves empanizadas $650 \mathrm{mg}$ en promedio.

El 38\% de los cereales de desayuno son altos en sodio y el $13 \%$ resultó por arriba de la meta regional de referencia de $630 \mathrm{mg}$ de sodio en $100 \mathrm{~g}$ de cereales de desayuno. Resultó un contenido promedio de sodio en esta categoría de 385 mg.

El $55 \%$ del snack resultó alto en sodio y el $23 \%$ con sodio mayor a la meta regional, siendo la meta de referencia $900 \mathrm{mg}$ de sodio en $100 \mathrm{~g}$ de snacks. Esta categoría resultó con 711 mg de sodio en promedio.

Los 35 productos de sopas como se consumen resultaron altos en sodio y 33 de estos formulados por arriba de la meta regional de $360 \mathrm{mg}$ de sodio en $100 \mathrm{~g}$. El promedio de sodio en sopas fue de $1472 \mathrm{mg}$.

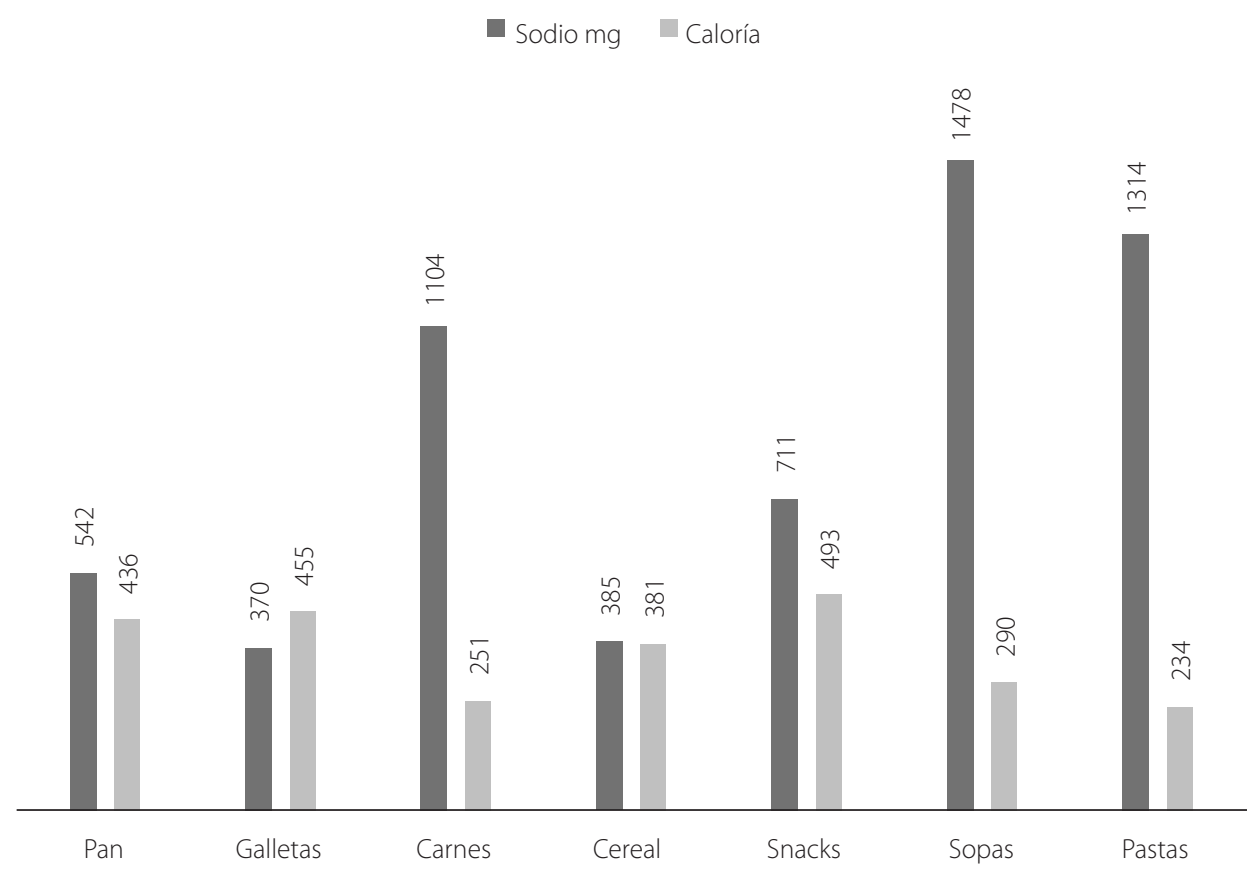

Figura 1. Productos alimenticios preenvasados según contenido promedio de sodio (mg) y de calorías en 100 gramos de siete categorías comercializados en El Salvador. 


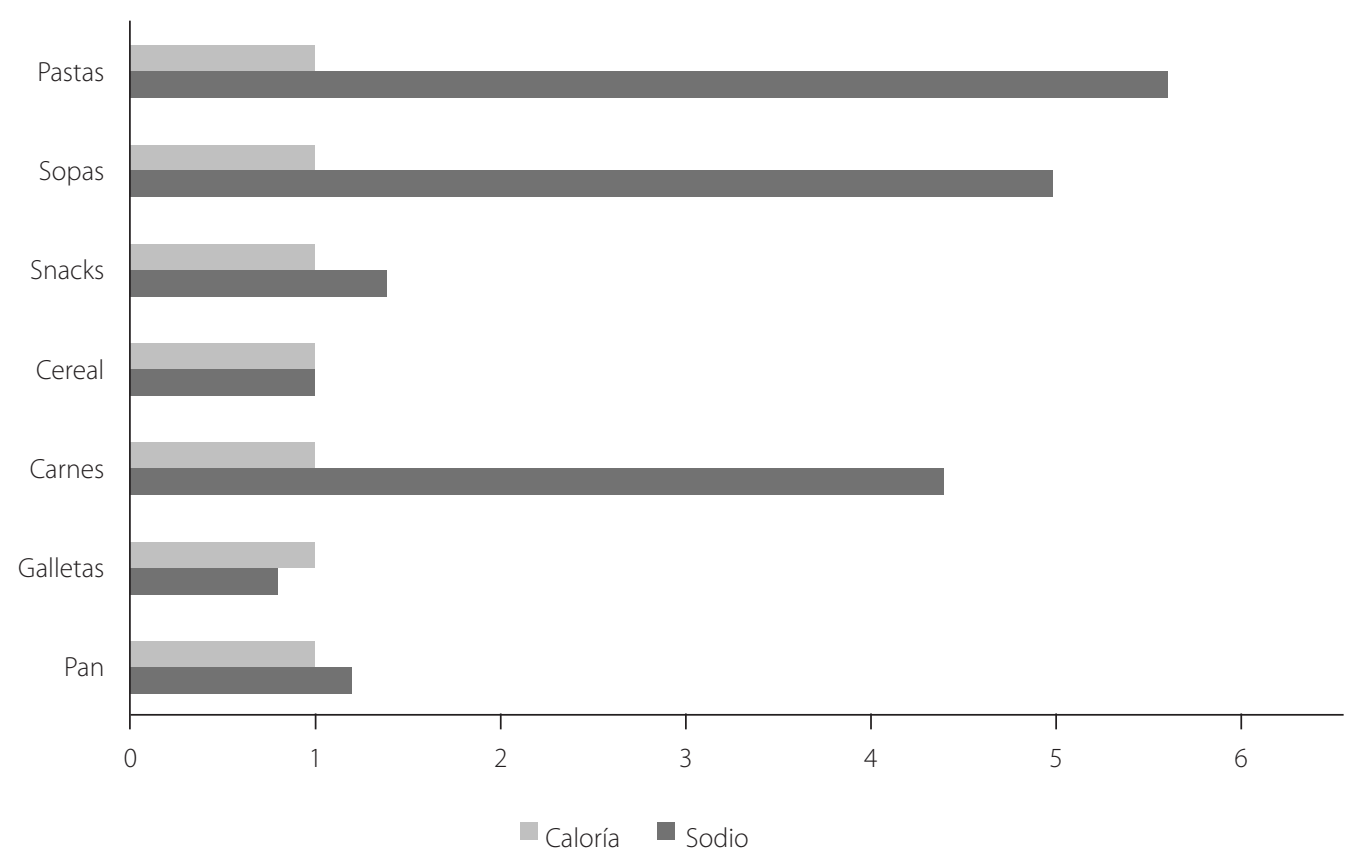

Figura 2. Relación sodio (mg) por caloría en productos alimenticios de siete categorías comercializados en El Salvador.

Las 27 de 28 pastas de larga duración tal como se consumen resultaron altas en sodio y 18 con contenido de sodio por arriba de meta regional de $640 \mathrm{mg}$ de sodio en $100 \mathrm{~g}$. El resultado promedio de sodio en pastas fue de $1314 \mathrm{mg}$.

\section{Discusión}

El estudio evaluó el contenido de sodio en productos alimenticios comercializados en El Salvador, encontrándose que más de la mitad de los productos analizados son altos en sodio según el perfil de nutrientes y menos de una cuarta parte de los productos están formulados industrialmente con contenido de sodio por arriba de las metas regionales. Al realizar el análisis por categorías, las sopas, pastas y carnes resultaron ser los productos con más alto contenido de sodio, seguidos del snack.

Una cuarta parte de los productos alimenticios incluidos en el estudio es de procedencia salvadoreña, el pan y snacks son las categorías con mayor porcentaje. El resto procede de otros países, principalmente de Estados Unidos y México. En la estrategia regional para la reducción del consumo de sal y sodio en Centroamérica y República Dominicana 2019-2025, una línea de acción importante es «Reducir el consumo de sodio en las formulaciones industriales de los productos procesados de las categorías de alimentos que más aporten a la ingesta de sodio» a través del trabajo en conjunto con el sector privado ${ }^{4}$. Para El Salvador, la posibilidad de un trabajo nacional en esta línea de acción es escasa, ya que la mayoría de pro- ductos se importan. Sin embargo, a través de una medida nacional se podría regular el ingreso de productos alimenticios a El Salvador, estableciendo estándares de contenido de sodio para autorizar su comercialización a través del registro sanitario.

Al analizar los resultados de la relación sodio/caloría se encontró que las categorías que más se desvían de la relación 1:1 son: las pastas, sopas y carnes que reflejan brechas amplias entre el promedio de sodio por arriba del promedio calórico. Las categorías pan, snack y cereales de desayuno con brechas sodio/calorías más estrechas, pero con promedio de sodio por arriba del promedio calórico y solamente la categoría de las galletas con sodio por debajo del promedio calórico a excepción de las galletas saladas.

Las brechas sodio/calorías están vinculadas a ingredientes declarados en la etiqueta general como sal, sal de cura, ajo en polvo, sabor limón sal, limón en polvo, sal yodada, condimento ajamonado, aditivos como nitrito de sodio, trifosfato de sodio, glutamato monosódico, eritorbato de sodio, inosinato de sodio, guanilato de sodio, bicarbonato de sodio, entre otros no específicos como potenciador del sabor, antioxidantes, mezclas de especies y otros ingredientes que adicionan sodio a los productos?

Como se reconoce en muchos análisis de la OMS, el patrón de consumo alimentario en el mundo presenta dietas altas en alimentos procesados, ultraprocesados y pobres en nutrientes. Los ingredientes que constituyen estas dietas presentan cantidades exorbitantes de sodio entre otros nutrientes $\operatorname{críticos}^{10}$. Según un estudio realiza- 
do en El Salvador, entre los productos más usados por los hogares salvadoreños se encuentran el pan dulce, las sopas deshidratadas, embutidos «carnes procesadas», snacks, y cereales de desayuno entre otros ${ }^{11}$. Según la evaluación del contenido de sodio, estos productos en su mayoría aportan cantidades excesivas de sodio a la dieta, ya que se registran para ser comercializados en supermercados, tiendas, cafeterías y mercados, siendo accesibles a la población y formando parte de los hábitos alimentarios que vinculan el consumo de sodio en la población salvadoreña.

La hipertensión arterial en adultos es la ENT de mayor prevalencia en el país afectando aproximadamente a 4 de 10 adultos, según la Encuesta Nacional de Enfermedades Crónicas del año 2014-2015, lo que constituye un grave problema de salud pública ${ }^{8}$. El Salvador aún no cuenta con una estrategia nacional para la reducción del consumo de sodio en la dieta, conociéndose que el componente nutricional más relacionado con las cifras de presión arterial elevada, aparte del exceso calórico, es el consumo de sodio.

El consumo adecuado de sodio debe ser de 1500 mg al día ${ }^{12}$. Como máximo, según situación actual a escala mundial, debe ser por debajo de $2000 \mathrm{mg}$ al día ${ }^{13}$, para una persona adulta sana con actividad moderada. Al relacionar el contenido de sodio que brindan a la dieta estos productos alimenticios (como «pastas, sopas, carnes, snacks»), se calcula que estarían aportando a un individuo entre el total o la mitad de las ingestas adecuadas de sodio en una porción consumida. Para los niños menores de 10 años, los aportes de sodio estarían superando las recomendaciones de ingestas adecuadas, así como en adultos mayores de 70 años ${ }^{12,13}$.

La respuesta de la presión arterial a la restricción de sodio varía entre individuos y se calcula que el $60 \%$ de los hipertensos son sensibles a la disminución de sodio. La población salvadoreña cada vez más se expone a consumir productos alimenticios altos en sodio. Por lo tanto, el consumo de estos productos tiene mayores implicaciones en la salud de pacientes con ENT que requieren dietas hiposódicas, ya que según el manejo individualizado estas dietas pueden ser: estrictas ( $<500 \mathrm{mg}$ sodio/día), moderadas (500-1000 mg sodio/día) o de restricción leve (3000 a 2000 mg sodio/día). Una sola porción estaría aportando más de lo recomendado en su tratamiento dietoterapéutico, además de estar limitando o complicando la respuesta del paciente a su tratamiento integral14-17.

Actualmente, los productos alimenticios no tienen regulaciones obligatorias sobre cantidad de sodio, solo es obligatorio declarar el contenido de sodio en la etiqueta nutricional de productos alimenticios previamente envasados que incluyan información nutricional en el envase ${ }^{18,19}$. Los resultados permiten observar que las metas regionales son flexibles ya que existe diferencia en la valoración de contenido de sodio. Al clasificar los productos por contenido de sodio, la mayoría han cumplido las metas regionales y, según el perfil de nutrientes, menos de la mitad de los productos cumple la relación sodio/caloría.

En las bases de datos se identificaron productos con registro sanitario que declararon $0 \%$ de sodio en la etiqueta nutricional, a pesar de que en su lista de ingredientes del etiquetado general se encontraba la sal yodada, bicarbonato de sodio, glutamato monosódico, sal de ajo, entre otros. Estos productos no se incluyeron en el estudio por declaraciones sodio imprecisas, que no correspondían a sus ingredientes. Sin embargo, los resultados de la investigación e interpretación se han realizado con base a las declaraciones de cada empresa cuando registra el producto.

Nuevos estudios de evaluación de contenido de sodio en productos alimenticios preenvasados deberían confirmar las declaraciones de las etiquetas con una submuestra que incluya análisis químico de sodio.

Estos resultados son importantes para El Salvador. De no haber medidas de carácter global sin existir acciones nacionales, se estaría manteniendo el patrón de consumo de sodio, ya advertido por la OPS/OMS/Instituto de Nutrición de Centro América y Panamá, que indica que la ingesta media diaria de sal en las Américas es superior a los 5 g 20,21,22. Responder a la problemática de la prevalencia de la hipertensión arterial en El Salvador requiere de medidas en salud pública más allá de medidas sanitarias de manejo integral de la enfermedad en diferentes niveles de atención en salud o del tratamiento farmacológico y dietético individual. También requiere de medidas sanitarias de carácter ambiental o regulatorias que permitan el ingreso de productos alimenticios al país de aquellos que cumplan estándares de contenido de sodio para su comercialización.

Reducir el consumo de sodio en la población precisa de acciones con la industria y otros sectores para implementación de medidas como reformulación de alimentos, la vigilancia alimentaria a nivel sanitario, regulaciones sanitarias, monitoreo del consumo de sodio en la población, etiquetado nutricional interpretativo para la población y promoción de una alimentación saludable en sectores claves ${ }^{23,24,25}$. 


\section{Conclusión}

Más de la mitad los productos alimenticios preenvasados comercializados en El Salvador son clasificados como altos en sodio, según el perfil de nutrientes y menos de una cuarta parte de estos, están formulados con sodio por arriba de las metas regionales para la formulación industrial, mostrando que las metas son flexibles, ya que existe diferencia en la valoración del excesivo contenido de sodio con relación al perfil de nutrientes.

Al evaluar el promedio de sodio por calorías en 100 gramos de cada producto se encontró que las pastas, sopas y carnes son los que más contenido de sodio registran por calorías seguidas de los snacks, pan, los cereales de desayuno y solo la categoría galletas resulto con promedio de sodio menor al promedio de calorías.

Tomando en consideración que, con respecto a los productos alimenticios estudiados, la industria nacional representa una minoría, cualquier gestión normalizadora conducente a la comercialización de productos alimenticios en El Salvador, deberá de considerar de manera efectiva los productos de importación.

\section{Agradecimiento}

Al Ministerio de Salud de El Salvador y al Instituto de Nutrición de Centro América y Panamá, por sus contribuciones al estudio.

\section{Financiamiento}

Financiamiento propio, con apoyo institucional en el tiempo y medios para la recolección de datos.

\section{Referencias bibliográficas}

1. Organización Mundial de la Salud. Enfermedades no transmisibles. Organización Mundial de la Salud. 2018. Fecha de consulta 1 de marzo de 2019. Disponible en: https://cutt.ly/qmHglAS

2. Feng J, Tan M, Yuan M, MacGregor G. Salt Reduction to Prevent Hypertension and Cardiovascular Disease. Journal of the American College of Cardiology Foundation. 2020; 75(6):632-647. DOI: 10.1016/j. jacc.2019.11.055

3. Organización Panamericana de la Salud/ OMS. Declaración de consenso del Consorcio "Cuídate de la sal". Organización Panamericana de la Salud. 2015. Fecha de consulta 19 de junio de 2019. Disponible: https://cutt.ly/8mHgnRP
4. Comisión Técnica de Enfermedades Crónicas y Cáncer. Estrategia regional para la reducción del consumo de sal y sodio en Centroamérica y República Dominicana 2019-2025. Instituto de nutrición de Centroamérica y Panamá. 2018. 32p. Disponible en: https://cutt.ly/7mHgRQy

5. Modelo de perfil de nutrientes de la Organización Panamericana de la Salud. Organización Panamericana de la Salud. Washinton, DC. 2016. Disponible en: https://cutt.ly/RmHgHsh

6. Plan estratégico nacional multisectorial para el abordaje integral de las enfermedades no transmisibles. Ministerio de Salud. San Salvador, El Salvador. 2017. Disponible en: https://cutt.ly/KmHg7TI

7. Lineamientos técnicos para el abordaje integral de la hipertensión arterial, diabetes mellitus y enfermedad renal crónica en el primer nivel de atención. Ministerio de Salud. San Salvador, El Salvador. 2021. Disponible en: https://cutt.ly/kmHhiTT

8. Ministerio de Salud. Encuesta Nacional de Enfermedades Crónicas no Transmisibles en población adulta de El Salvador ENECAELS 2015. El Salvador. Instituto Nacional de Salud. 2017. 252p. Disponible en: https://cutt.ly/jmHhfSU

9. Alimentos y Bebidas Procesadas. Aditivos Alimentarios. El Salvador. 21 de junio de 2012.

10. Campbell N, Legowski B, Legetic B, Nilson $B$, L'Abbe M. Inaugural maximum values for sodium in processed food product in the Americas. The Journal of Clinical Hypertension. 2015; 17(8):611-613. DOI: 10.1111/jch.12553

11. Menchú MT, Méndez H. Análisis de la Situación Alimentaria en Guatemala. Guatemala. Instituto de Nutrición de Centroamérica y Panamá INCAP. 2011. 57p. Disponible en: https://cutt.ly/HmHhmOf

12. Menchú MT, Torún B, Elías LG. Recomendaciones Dietéticas Diarias del INCAP. 2da Edición. Guatemala. INCAP Ed; 2012. 222p.

13. Ingesta de sodio en adultos y niños. Organización mundial de la Salud. Ginebra, Suiza. 2013. Disponible en: https://cutt.ly/QmHhASV

14. Atlas mundial sobre prevención y control de enfermedades cardiovasculares. Organización mundial de la Salud. Ginebra, Suiza. 2011. Disponible en: https://cutt.ly/UmHhM4N

15. Organización Mundial de la Salud. Reducir la ingesta de sodio para reducir la tensión arterial y el riesgo de enfermedades cardiovasculares en adultos. Organización Mundial de la Salud. 2020. Fecha de 
consulta: 1 de febrero de 2020. Disponible en: https://cutt.ly/amHh5U9

16. Lineamientos Técnicos Para La Prescripción De Dietas En Hospitales De La RIISS. Ministerio de Salud. San Salvador, El Salvador. 2014. Disponible en: https://cutt.ly/SmHjtZs

17. Ortega R, Jiménez A, Perea J, Cuadrado E, López A. Pautas nutricionales en prevención y control de la hipertensión arterial Nutrición Hospitalaria. Nutrición Hospitalaria. 2016.33 (4); 53-58.

DOl: 10.20960/nh.347

18. Etiquetado Nutricional de productos alimenticios preenvasados para consumo humano para la población a partir de 3 años de edad. El Salvador. 23 de diciembre de 2011.

19. Etiquetado general de los alimentos previamente envasados. El Salvador. 21 de junio de 2012.

20. Organización Mundial de la Salud. Es hora de actuar: informe de la Comisión Independiente de Alto Nivel de la OMS sobre Enfermedades No Transmisibles. Ginebra. Organización Mundial de la Salud. 2018. 48p. Disponible en: https://cutt.ly/amHjfRx

21. SHAKE menos sal, más salud. Guía para el establecimiento de metas y cronologías para reducir el contenido de sal de los alimentos. Organización Panamericana de la Salud. Washington, D.C. 2018. Disponible en: https://cutt.ly/ImHjvk4

22. López P, Pérez W. Situación del consumo de sal y sodio en Centroamérica y República Dominicana. Guatemala. Instituto de Nutrición de Centroamérica y Panamá, INCAP. 2018. 5 p. Disponible en: https://cutt.ly/BmHjE6T

23. Organización de las Naciones Unidas para la Alimentación y la Agricultura. El futuro de la alimentación y la agricultura, Tendencias y Desafíos. Roma. FAO. 2017. 52 p. Disponible en: http://www.fao.org/3/i6881s/i6881s.pdf

24. Rapallo R, Rivera R. Nuevos patrones alimentarios, más desafíos para los sistemas alimentarios. Santiago de Chile. FAO. 2019. 25 p. Disponible en: http://www.fao.org/3/ca5449es/ca5449es. pdf

25. Arguelles J, Nuñez P, Perrillan C. Consumo excesivo de sal e hipertensión arterial: Implicaciones para la salud pública. Revista mexicana de trastornos alimentarios. 2018; 9(1):119-128. DOI: 10.22201/ fesi.20071523e.2018.1.466 